\title{
Bounds of the logarithmic mean
}

\author{
Shigeru Furuichi ${ }^{*}$ and Kenjiro Yanagi ${ }^{2}$
}

"Correspondence:

furuichi@chs.nihon-u.ac.jp

'Department of Information

Science, College of Humanities and

Sciences, Nihon University, 3-25-40,

Sakurajyousui, Setagaya-ku, Tokyo,

156-8550, Japan

Full list of author information is

available at the end of the article

\begin{abstract}
We give tight bounds for the logarithmic mean. We also give new Frobenius norm inequalities for two positive semidefinite matrices. In addition, we give some matrix inequalities on the matrix power mean.
\end{abstract}

MSC: $15 \mathrm{~A} 39 ; 15 \mathrm{~A} 45$

Keywords: logarithmic mean; matrix mean; Frobenius norm and inequality

\section{Introduction}

In this short paper, we study the bounds of the logarithmic mean which is defined by

$$
L(a, b) \equiv \frac{a-b}{\log a-\log b}=\int_{0}^{1} a^{v} b^{1-v} d v, \quad a \neq b
$$

for two positive numbers $a$ and $b$. (We conventionally define $L(a, b)=a$ if $a=b$.) In the paper [1], the following relations were shown:

$$
\sqrt{a b} \leq L(a, b) \leq\left(\frac{a^{1 / 3}+b^{1 / 3}}{2}\right)^{3}, \quad a, b>0 .
$$

We now have the following lemma.

Lemma 1.1 For $a, b>0$, we have

$$
L(a, b) \leq\left(\frac{a^{1 / 3}+b^{1 / 3}}{2}\right)^{3} \leq \frac{2}{3} \sqrt{a b}+\frac{1}{3} \frac{a+b}{2} .
$$

Proof The second inequality of (3) can be proven easily. Indeed, we put $f(t) \equiv \frac{2}{3} t^{3}+\frac{1}{6}(1+$ $\left.t^{6}\right)-\frac{1}{8}\left(1+t^{2}\right)^{3}$. Then we have $f^{\prime}(t)<0$ for $0<t<1$ and $f^{\prime}(t)>0$ for $t>1$. Thus we have $f(t) \geq f(1)=0$ for $t>0$.

The first inequality of (3) refines the inequality

$$
L(a, b) \leq \frac{2}{3} \sqrt{a b}+\frac{1}{3} \frac{a+b}{2}, \quad a, b>0
$$

which is known as the classical Pólya inequality $[2,3]$.

Throughout this paper, we use the notation $M(n, \mathbb{C})$ as the set of all $n \times n$ matrices on the complex field $\mathbb{C}$. We also use the notation $M_{+}(n, \mathbb{C})$ as the set of all $n \times n$ positive semidefinite matrices. Here, $A \in M_{+}(n, \mathbb{C})$ means we have $\langle\phi|A| \phi\rangle \geq 0$ for any vector $|\phi\rangle \in \mathbb{C}^{n}$. For

\section{Springer}

๑2013 Furuichi and Yanagi; licensee Springer. This is an Open Access article distributed under the terms of the Creative Commons Attribution License (http://creativecommons.org/licenses/by/2.0), which permits unrestricted use, distribution, and reproduction in any medium, provided the original work is properly cited. 
$A \in M(n, \mathbb{C})$, the Frobenius norm (Hilbert-Schmidt norm) $\|\cdot\|_{F}$ is defined by

$$
\|A\|_{F} \equiv\left(\sum_{i, j=1}^{n}\left|a_{i j}\right|^{2}\right)^{1 / 2}=\left(\operatorname{Tr}\left[A^{*} A\right]\right)^{1 / 2} .
$$

In the paper [2], the following norm inequality was shown.

Theorem 1.2 ([2]) For $A, B \in M_{+}(n, \mathbb{C}), X \in M(n, \mathbb{C})$ and the Frobenius norm $\|\cdot\|_{F}$, we have

$$
\left\|\int_{0}^{1} A^{v} X B^{1-v} d v\right\|_{F} \leq \frac{1}{3}\left\|2 A^{1 / 2} X B^{1 / 2}+\frac{A X+X B}{2}\right\|_{F} .
$$

From Lemma 1.1, we have the following proposition.

Proposition 1.3 For $A, B \in M_{+}(n, \mathbb{C}), X \in M(n, \mathbb{C})$ and the Frobenius norm $\|\cdot\|_{F}$, we have

$$
\begin{aligned}
\left\|\int_{0}^{1} A^{v} X B^{1-v} d v\right\|_{F} & \leq\left\|\frac{A X+3 A^{1 / 3} X B^{2 / 3}+3 A^{2 / 3} X B^{1 / 3}+X B}{8}\right\|_{F} \\
& \leq \frac{1}{3}\left\|2 A^{1 / 2} X B^{1 / 2}+\frac{A X+X B}{2}\right\|_{F} .
\end{aligned}
$$

To the first author's best knowledge, the first inequality in Proposition 1.3 was suggested in [4].

This proposition can be proven in a similar way to the proof of Theorem 1.2 (or the proof of Theorem 2.2 which will be given in the next section), and this refines inequality (6) shown in [2].

\section{Lower bound of the logarithmic mean}

The following inequalities were given in [5]. Hiai and Kosaki gave the norm inequalities for Hilbert space operators in [5]. See also [6,7]. Here we give them as a matrix setting to unify the description of this paper.

Theorem 2.1 ([5]) For $A, B \in M_{+}(n, \mathbb{C}), X \in M(n, \mathbb{C}), m \geq 1$ and every unitarily invariant norm ||$\cdot \mid \|$, we have

$$
\left\|\int_{0}^{1} A^{v} X B^{1-v} d \nu\right\| \geq \frac{1}{m}\left\|\sum_{k=1}^{m} A^{k /(m+1)} X B^{(m+1-k) /(m+1)}\right\| \geq\left\|A^{1 / 2} X B^{1 / 2}\right\| .
$$

The Frobenius norm is one of unitarily invariant norms. We give the refinement of the lower bound of the first inequality above for the Frobenius norm. That is, we have the following inequalities.

Theorem 2.2 For $A, B \in M_{+}(n, \mathbb{C}), X \in M(n, \mathbb{C}), m \geq 1$ and the Frobenius norm $\|\cdot\|_{F}$, we have

$$
\begin{aligned}
\left\|\int_{0}^{1} A^{v} X B^{1-v} d v\right\|_{F} & \geq \frac{1}{m}\left\|\sum_{k=1}^{m} A^{(2 k-1) / 2 m} X B^{(2 m-(2 k-1)) / 2 m}\right\|_{F} \\
& \geq \frac{1}{m}\left\|\sum_{k=1}^{m} A^{k /(m+1)} X B^{(m+1-k) /(m+1)}\right\|_{F} \geq\left\|A^{1 / 2} X B^{1 / 2}\right\|_{F} .
\end{aligned}
$$


To prove Theorem 2.2, we need a few lemmas.

Lemma 2.3 Let $u, v, w$ be nonnegative integers such that $w \geq u$, and let $x$ be a positive real number. Then we have

$$
x^{u}\left(1-x^{v}\right)+x^{w}\left(x^{v}-1\right) \geq 0
$$

Proof It is trivial for the case $x=1$ or $v=0$. We prove for the case $x \neq 1$ and $v \neq 0$. In addition, for the case that $u=w$, the equality holds. Thus we may assume $w>u$ and $v \geq 1$. Then the lemma can be proven in the following way.

$$
\begin{aligned}
x^{u}\left(1-x^{v}\right)+x^{w}\left(x^{\nu}-1\right) & =\left(x^{\nu}-1\right)\left(x^{w}-x^{u}\right)=x^{u}\left(x^{v}-1\right)\left(x^{w-u}-1\right) \\
& =x^{u}(x-1)^{2}\left(x^{\nu-1}+x^{\nu-2}+\cdots+1\right)\left(x^{w-u-1}+x^{w-u-2}+\cdots+1\right) \\
& \geq 0 .
\end{aligned}
$$

Lemma 2.4 For a positive real number $x$ and a natural number $m$, we have

$$
\sum_{k=1}^{m} x^{(2 k-1)(m+1)} \geq \sum_{k=1}^{m} x^{2 k m}
$$

Proof For the case $x=1$, the equality holds. So we prove this lemma for $x \neq 1$. If $m$ is an odd number, then we have $m=2\lfloor m / 2\rfloor+1$. Since we then have $m+1=2(\lfloor m / 2\rfloor+1)$ and $2(\lfloor m / 2\rfloor+1)-1=m$, we have

$$
\{2(\lfloor m / 2\rfloor+1)-1\}(m+1)=2(\lfloor m / 2\rfloor+1) m
$$

If we put $\tilde{k}=\lfloor m / 2\rfloor+1$, then the above means $(2 \tilde{k}-1)(m+1)=2 \tilde{k} m$. Then the difference of the $\tilde{k}$ th term of the both sides in inequality (9) is equal to 0 . For the case that $m$ is an even number, it never happens that the difference of the $\tilde{k}$ th term of the both sides in inequality (9) is equal to 0 . Therefore we have

$$
\sum_{k=1}^{m} x^{(2 k-1)(m+1)}-\sum_{k=1}^{m} x^{2 k m}=\sum_{l=1}^{\lfloor m / 2\rfloor}\left\{x^{a_{l}}\left(1-x^{b_{l}}\right)+x^{c_{l}}\left(x^{b_{l}}-1\right)\right\},
$$

where $a_{l}=(2 l-1)(m+1), b_{l}=m-(2 l-1)$ and $c_{l}=2\{m-(l-1)\} m$ for $l=1,2, \ldots,\lfloor m / 2\rfloor$. Here we have $c_{l}-a_{l}=\{m-(2 l-1)\}(2 m+1) \geq 0$, whenever $b_{l} \geq 0$. By Lemma 2.3, if $b_{l} \geq 0$, then we have $x^{a_{l}}\left(1-x^{b_{l}}\right)+x^{c_{l}}\left(x^{b_{l}}-1\right) \geq 0$. Thus the proof of this lemma is completed.

If we put $t=x^{2 m(m+1)}>0$, then we have

$$
\sum_{k=1}^{m} t^{(2 k-1) / 2 m} \geq \sum_{k=1}^{m} t^{k /(m+1)} \quad(t>0, m \in \mathbb{N})
$$

which implies

$$
\sum_{k=1}^{m} a^{(2 k-1) / 2 m} b^{(2 m-(2 k-1)) / 2 m} \geq \sum_{k=1}^{m} a^{k /(m+1)} b^{(m+1-k) /(m+1)} \quad(a, b>0, m \in \mathbb{N}) .
$$


We then have the following lemma.

Lemma 2.5 For $a, b>0$ and $m \geq 1$, we have

$$
L(a, b) \geq \frac{1}{m} \sum_{k=1}^{m} a^{(2 k-1) / 2 m} b^{(2 m-(2 k-1)) / 2 m} \geq \frac{1}{m} \sum_{k=1}^{m} a^{k /(m+1)} b^{(m+1-k) /(m+1)} \geq \sqrt{a b} .
$$

Proof The second inequality follows by inequality (11). We use the famous inequality $(x-1) / \log x \geq \sqrt{x}$ for $x>0$. We put $x=t^{1 / m}$ in this inequality. Then we have, for $t>0$,

$$
\frac{t-1}{\log t} \geq \frac{t^{(2 m-1) / 2 m}+t^{(2 m-3) / 2 m}+\cdots+t^{1 / 2 m}}{m}
$$

which implies the first inequality. The third inequality can be proven by the use of the arithmetic mean-geometric mean inequality. Thus the proof of this lemma is completed.

We give some basic properties of the right-hand side of inequality (13) in the Appendix.

Proof of Theorem 2.2 It is known that the Frobenius norm inequality immediately follows from the corresponding scalar inequality [6]. However, we give here an elementary proof for the convenience of the readers. Let $U^{*} A U=D_{1}=\operatorname{diag}\left\{\alpha_{1}, \ldots, \alpha_{n}\right\}$ and $V^{*} B V=D_{2}=$ $\operatorname{diag}\left\{\beta_{1}, \ldots, \beta_{n}\right\}$. Then $\alpha_{1}, \ldots, \alpha_{n} \geq 0$ and $\beta_{1}, \ldots, \beta_{n} \geq 0$. We put $U^{*} X V \equiv Y=\left(y_{i j}\right)$. By the first inequality of (12), we have

$$
\begin{aligned}
& \frac{1}{m}\left\|\sum_{k=1}^{m} A^{(2 k-1) / 2 m} X B^{(2 m-(2 k-1)) / 2 m}\right\|_{F} \\
& =\frac{1}{m}\left\|\sum_{k=1}^{m} U D_{1}^{(2 k-1) / 2 m} U^{*} X V D_{2}^{(2 m-(2 k-1)) / 2 m} V^{*}\right\|_{F} \\
& =\frac{1}{m}\left\|\sum_{k=1}^{m} D_{1}^{(2 k-1) / 2 m} Y D_{2}^{(2 m-(2 k-1)) / 2 m}\right\|_{F} \\
& =\frac{1}{m}\left\|\left(\begin{array}{ccc}
\sum_{k=1}^{m} \alpha_{1}^{(2 k-1) / 2 m} y_{11} \beta_{1}^{(2 m-(2 k-1)) / 2 m} & \cdots & \sum_{k=1}^{m} \alpha_{1}^{(2 k-1) / 2 m} y_{1 n} \beta_{n}^{(2 m-(2 k-1)) / 2 m} \\
\vdots & \ddots & \vdots \\
\sum_{k=1}^{m} \alpha_{n}^{(2 k-1) / 2 m} y_{n 1} \beta_{1}^{(2 m-(2 k-1)) / 2 m} & \cdots & \sum_{k=1}^{m} \alpha_{n}^{(2 k-1) / 2 m} y_{n n} \beta_{n}^{(2 m-(2 k-1)) / 2 m}
\end{array}\right)\right\|_{F} \\
& =\left\{\sum_{i, j=1}^{n}\left(\frac{1}{m} \sum_{k=1}^{m} \alpha_{i}^{(2 k-1) / 2 m} \beta_{j}^{(2 m-(2 k-1)) / 2 m}\right)^{2}\left|y_{i j}\right|^{2}\right\}^{1 / 2} \\
& \leq\left\{\sum_{i, j=1}^{n}\left(\int_{0}^{1} \alpha_{i}^{v} \beta_{j}^{1-v} d v\right)^{2}\left|y_{i j}\right|^{2}\right\}^{1 / 2}=\left\|\int_{0}^{1} D_{1}^{v} Y D_{2}^{1-v} d v\right\|_{F}=\left\|U \int_{0}^{1} D_{1}^{v} Y D_{2}^{1-v} d \nu V^{*}\right\|_{F} \\
& =\left\|\int_{0}^{1} U D_{1}^{v} U^{*} X V D_{2}^{1-v} V^{*} d v\right\|_{F}=\left\|\int_{0}^{1} A^{v} X B^{1-v} d v\right\|_{F} \text {. }
\end{aligned}
$$

Applying inequality (11), we have the second inequality of (7) in a similar way. The third inequality holds due to Theorem 2.1 (or the third inequality of (12)). 


\section{Upper bound of the logarithmic mean}

In the paper [5], the following norm inequalities were also given for Hilbert space operators. Here we give them for matrices, as we mentioned in the beginning of Section 2.

Theorem 3.1 ([5]) For $A, B \in M_{+}(n, \mathbb{C}), X \in M(n, \mathbb{C}), m \geq 2$ and every unitarily invariant norm $\|\cdot \mid\|$, we have

$$
\left\|\int_{0}^{1} A^{v} X B^{1-v} d v\right\| \leq \frac{1}{m}\left\|\sum_{k=0}^{m-1} A^{k /(m-1)} X B^{(m-1-k) /(m-1)}\right\| \leq \frac{1}{2}\|A X+X B\| .
$$

We also give an improved upper bound of the logarithmic mean on Theorem 3.1 above, only for the Frobenius norm. Namely, we can prove the following inequalities in a similar way to the proof of Theorem 2.2, by the use of scalar inequalities which will be given in Lemma 3.4.

Theorem 3.2 For $A, B \in M_{+}(n, \mathbb{C}), X \in M(n, \mathbb{C}), m \geq 2$ and the Frobenius norm $\|\cdot\|_{F}$, we have

$$
\begin{aligned}
\left\|\int_{0}^{1} A^{v} X B^{1-v} d v\right\|_{F} & \leq \frac{1}{m}\left\|\sum_{k=0}^{m} A^{k / m} X B^{(m-k) / m}-\frac{1}{2}(A X+X B)\right\|_{F} \\
& \leq \frac{1}{m}\left\|\sum_{k=0}^{m-1} A^{k /(m-1)} X B^{(m-1-k) /(m-1)}\right\|_{F} \leq \frac{1}{2}\|A X+B X\|_{F} .
\end{aligned}
$$

To prove Theorem 3.2, we need to prove the following lemmas.

Lemma 3.3 For $x>0$ and $m \geq 2$, we have

$$
\sum_{k=1}^{m-1}\left(x^{k m}-x^{k(m-1)}\right) \geq \frac{x^{m(m-1)}-1}{2} \quad(x>0) .
$$

Proof For $m \geq 2$, we calculate

$$
\begin{aligned}
& \sum_{k=1}^{m-1}\left(x^{k m}-x^{k(m-1)}\right)-\frac{x^{m(m-1)}-1}{2} \\
& \quad=\frac{1}{2}\left(x^{m}+1\right)\left\{\left(x^{m}\right)^{m-2}+\cdots+x^{m}+1\right\}-x^{m-1}\left\{\left(x^{m-1}\right)^{m-2}+\cdots+x^{m-1}+1\right\} \\
& \quad \geq x^{m / 2}\left\{\left(x^{m}\right)^{m-2}+\cdots+x^{m}+1\right\}-x^{m-1}\left\{\left(x^{m-1}\right)^{m-2}+\cdots+x^{m-1}+1\right\} .
\end{aligned}
$$

Here, we put $y \equiv x^{1 / 2}>0$, then we have

$$
\begin{aligned}
(16)= & y^{m}\left\{\left(y^{2 m}\right)^{m-2}+\cdots+y^{2 m}+1\right\}-y^{2(m-1)}\left\{\left(y^{2(m-1)}\right)^{m-2}+\cdots+y^{2(m-1)}+1\right\} \\
= & y^{m}\left(1-y^{m-2}\right)+y^{3 m}\left(1-y^{m-4}\right)+y^{5 m}\left(1-y^{m-6}\right)+\cdots \\
& +y^{2(m-1)(m-3)}\left(y^{m-6}-1\right)+y^{2(m-1)(m-2)}\left(y^{m-4}-1\right)+y^{2(m-1)^{2}}\left(y^{m-2}-1\right) \\
= & \sum_{l=1}^{\lfloor m / 2\rfloor}\left\{y^{p_{l}}\left(1-y^{r_{l}}\right)+y^{q_{l}}\left(y^{r_{l}}-1\right)\right\} \geq 0,
\end{aligned}
$$


where $p_{l}=(2 l-1) m, q_{l}=2(m-1)(m-l)$ and $r_{l}=m-2 l$. The last inequality follows from Lemma 2.3 , because we have $q_{l}-p_{l}=(2 m-1)(m-2 l) \geq$ whenever $r_{l} \geq 0$.

Lemma 3.4 For $a, b>0$ and $m \geq 2$, we have

$$
\begin{aligned}
L(a, b) & \leq \frac{1}{m}\left\{\sum_{k=0}^{m} a^{k / m} b^{(m-k) / m}-\left(\frac{a+b}{2}\right)\right\} \\
& \leq \frac{1}{m} \sum_{k=0}^{m-1} a^{k /(m-1)} b^{(m-1-k) /(m-1)} \leq \frac{a+b}{2} .
\end{aligned}
$$

Proof To prove the first inequality, we have only to prove the following inequality:

$$
\frac{t-1}{\log t} \leq \frac{1}{m}\left\{\left(t+t^{(m-1) / m}+t^{(m-2) / m}+\cdots+t^{1 / m}+1\right)-\left(\frac{t+1}{2}\right)\right\} \quad(t>0) .
$$

Inequality (19) can be proven by putting $x \equiv t^{1 / m}>0$ in the famous inequality $(x-1) /$ $\log x \leq(x+1) / 2$ for $x>0$.

To prove the second inequality of (18), it is sufficient to prove inequality (15) which follows from Lemma 3.3. We obtain actually the second inequality of (18) by putting $t=x^{m(m-1)}>0$ in inequality (15), and then putting $t=a / b$.

To prove the third inequality of (18), it is sufficient to prove the following inequality:

$$
t^{m-1}+\cdots+t+1 \leq \frac{m\left(t^{m-1}+1\right)}{2} \quad(t>0)
$$

This inequality can be proven by the induction on $m$. Indeed, we assume that the inequality (20) holds for some $m$. Then we add $t^{m}>0$ to both sides of inequality (20). Then we have

$$
t^{m}+t^{m-1}+\cdots+t+1 \leq \frac{m\left(t^{m-1}+1\right)}{2}+t^{m}
$$

Therefore we have only to prove the inequality

$$
\frac{m\left(t^{m-1}+1\right)}{2}+t^{m} \leq \frac{(m+1)\left(t^{m}+1\right)}{2} \quad(t>0)
$$

which is equivalent to the inequality

$$
(m-1) t^{m}-m t^{m-1}+1 \geq 0 \quad(t>0) .
$$

We put $f_{m}(t) \equiv(m-1) t^{m}-m t^{m-1}+1$. Then we can prove $f_{m}(t) \geq f_{m}(1)=0$ by elementary calculations. Thus inequality (20) holds for $m+1$.

We give some basic properties of the right-hand side of inequality (19) in the Appendix.

\section{Matrix inequalities on the geometric mean}

Using Lemma 1.1, Lemma 2.5 and Lemma 3.4, we have following Proposition 4.1, Proposition 4.2 and Proposition 4.3, respectively. 
Proposition 4.1 For $A, B \in M_{+}(n, \mathbb{C})$, we have

$$
\int_{0}^{1} A \#_{\nu} B d v \leq \frac{1}{4}\left\{\frac{A+B}{2}+\frac{3}{2}\left(A \#_{2 / 3} B+A \#_{1 / 3} B\right)\right\} \leq \frac{1}{2}\left(\frac{A+B}{2}+2 A \#_{1 / 2} B\right),
$$

where $A \#_{\nu} B \equiv A^{1 / 2}\left(A^{-1 / 2} B A^{-1 / 2}\right)^{v} A^{1 / 2}(0 \leq v \leq 1)$ is $v$-weighted geometric mean introduced in $[8]$.

Proposition 4.2 For $A, B \in M_{+}(n, \mathbb{C})$ and $m \geq 1$, we have

$$
\int_{0}^{1} A \#_{\nu} B d \nu \geq \frac{1}{m} \sum_{k=1}^{m} A \#_{(2 k-1) / 2 m} B \geq \frac{1}{m} \sum_{k=1}^{m} A \#_{k /(m+1)} B \geq A \#_{1 / 2} B .
$$

Proposition 4.3 For $A, B \in M_{+}(n, \mathbb{C})$ and $m \geq 2$, we have

$$
\int_{0}^{1} A \#_{\nu} B d \nu \leq \frac{1}{m}\left(\sum_{k=0}^{m} A \#_{k / m} B-\frac{A+B}{2}\right) \leq \frac{1}{m} \sum_{k=0}^{m-1} A \#_{k /(m-1)} B \leq \frac{A+B}{2} .
$$

We give the proof of Proposition 4.3. Proposition 4.1 and Proposition 4.2 are also proven in a similar way, using Lemma 1.1 and Lemma 2.5. In addition, by using the notion of the representing function $f_{m}(x)=1 m x$ for the operator mean $m$, it is well known [8] that $f_{m}(x) \leq f_{n}(x)$ holds for $x>0$ if and only if $A m B \leq A n B$ holds for all positive operators $A$ and $B$. However, we give an elementary proof for the convenience of the readers.

Proof of Proposition 4.3 Since $T \equiv A^{-1 / 2} B A^{-1 / 2} \geq 0$, there exists a unitary matrix $U$ such that $U^{*} T U=D \equiv \operatorname{diag}\left\{\lambda_{1}, \ldots, \lambda_{n}\right\}$. Then $\lambda_{1}, \ldots, \lambda_{n} \geq 0$. From Lemma 3.4, for $i=1, \ldots, n$, we have

$$
\int_{0}^{1} \lambda_{i}^{\nu} d \nu \leq \frac{1}{m}\left\{\sum_{k=0}^{m} \lambda_{i}^{k / m}-\left(\frac{\lambda_{i}+1}{2}\right)\right\} \leq \frac{1}{m} \sum_{k=0}^{m-1} \lambda_{i}^{k /(m-1)} \leq \frac{\lambda_{i}+1}{2} .
$$

Thus we have

$$
\int_{0}^{1} D^{\nu} d \nu \leq \frac{1}{m}\left\{\sum_{k=0}^{m} D^{k / m}-\left(\frac{D+I}{2}\right)\right\} \leq \frac{1}{m} \sum_{k=0}^{m-1} D^{k /(m-1)} \leq \frac{D+I}{2} .
$$

Multiplying $U$ and $U^{*}$ to both sides, we have

$$
\int_{0}^{1} T^{v} d v \leq \frac{1}{m}\left\{\sum_{k=0}^{m} T^{k / m}-\left(\frac{T+I}{2}\right)\right\} \leq \frac{1}{m} \sum_{k=0}^{m-1} T^{k /(m-1)} \leq \frac{T+I}{2} .
$$

Inserting $T \equiv A^{-1 / 2} B A^{-1 / 2}$ and then multiplying two $A^{1 / 2}$ to all sides from both sides, we obtain the result.

Closing this section, we give another matrix inequalities by the use of another lower bound of the logarithmic mean. As another lower bound of the logarithmic mean, the 
following inequalities are known:

$$
\frac{t-1}{\log t} \geq \frac{t+t^{1 / 3}}{1+t^{1 / 3}} \geq \sqrt{t}, \quad t>0, t \neq 1
$$

The proofs of the above inequalities are not so difficult (they can be done by putting $x=$ $t^{1 / 3}>0$ and $x=t^{1 / 6}>0$ ), here we omit them. From inequalities (21), we have

$$
\int_{0}^{1} t^{\nu} d v \geq t^{2 / 3}-t^{1 / 3}+2\left(t^{-1 / 3}+1\right)^{-1} \geq \sqrt{t}, \quad t>0, t \neq 1
$$

Inequalities (22) imply the following result in a similar way to the proof of Proposition 4.3.

Proposition 4.4 For $A, B \in M_{+}(n, \mathbb{C})$, we have

$$
\int_{0}^{1} A \#_{\nu} B d \nu \geq A \#_{2 / 3} B-A \#_{1 / 3} B+2\left\{\left(A \#_{1 / 3} B\right)^{-1}+A^{-1}\right\}^{-1} \geq A \#_{1 / 2} B .
$$

\section{Comments}

Proposition 6.2 given in the Appendix shows that our upper bound is tighter than the standard upper bound for the case $t>1$ and our lower bound is tighter than the standard lower bound for any $t>0$. In addition, our lower bound $\alpha_{m}(t)$ of the logarithmic mean $L(t, 1)$ is tighter than the lower bound $\sqrt{t}$ given by Lin in [1] for $m \geq 1$. However, it may be a difficult problem to find the minimum $m \in \mathbb{N}$ such that $\beta_{m}(t) \leq\left(\left(t^{1 / 3}+1\right) / 2\right)^{3}$ for any $t>0$. The right-hand side of the above inequality is the upper bound given by Lin in [1]. (See inequalities (2).)

\section{Appendix}

Here we note some basic properties of the following scalar sums:

$$
\alpha_{m}(t) \equiv \frac{1}{m} \sum_{k=1}^{m} t^{(2 k-1) /(2 m)}, \quad \beta_{m}(t) \equiv \frac{1}{m}\left(\sum_{k=0}^{m} t^{k / m}-\frac{t+1}{2}\right)
$$

for $t>0$.

Proposition 6.1 For any $t>0$, we have following properties:

(i) $\alpha_{m}(t) \leq \alpha_{m+1}(t)$.

(ii) $\beta_{m+1}(t) \leq \beta_{m}(t)$.

(iii) $\alpha_{m}(t)$ and $\beta_{m}(t)$ converges to $L(t, 1)$ as $m \rightarrow \infty$. In addition, we have $\alpha_{m}(t) \leq \beta_{m}(t)$.

Proof We prove (i)-(iii) for $t \neq 1$, since it is trivial for the case $t=1$.

(i) Since

$$
\alpha_{m}(t)=\frac{t^{1 /(2 m)}(t-1)}{m\left(t^{1 / m}-1\right)}
$$

for $t \neq 1$, we prove

$$
\frac{\alpha_{m+1}(t)}{\alpha_{m}(t)}=\frac{m t^{1 /(2(m+1))}\left(t^{1 / m}-1\right)}{(m+1) t^{1 /(2 m)}\left(t^{1 /(m+1)}-1\right)}>1
$$


for $t>0$ and $t \neq 1$. We first prove the case $t>1$. Then we put $s \equiv t^{1 /(2 m(m+1))}$ and

$$
f_{m}(s) \equiv m\left(s^{2 m+2}-1\right)-(m+1)\left(s^{2 m+1}-s\right) \quad(s>1) .
$$

By elementary calculations, we have $f_{m}(s)>f_{m}(1)=0$, which implies

$$
m s^{m}\left(s^{2 m+2}-1\right)>(m+1) s^{m+1}\left(s^{2 m}-1\right) .
$$

We can prove similarly

$$
m s^{m}\left(s^{2 m+2}-1\right)<(m+1) s^{m+1}\left(s^{2 m}-1\right)
$$

for the case $0<s<1$.

(ii) Since

$$
\beta_{m}(t)=\frac{\left(t^{1 / m}+1\right)(t-1)}{2 m\left(t^{1 / m}-1\right)}
$$

for $t \neq 1$, we prove

$$
\frac{\beta_{m+1}(t)}{\beta_{m}(t)}=\frac{m\left(t^{1 /(m+1)}+1\right)\left(t^{1 / m}-1\right)}{(m+1)\left(t^{1 / m}+1\right)\left(t^{1 /(m+1)}-1\right)}<1
$$

for $t>0$ and $t \neq 1$. We first prove the case $t>1$. Then we put $r \equiv t^{1 /(m(m+1))}$ and

$$
g_{m}(r) \equiv r^{2 m+1}-(2 m+1) r^{m+1}+(2 m+1) r^{m}-1 \quad(r>1) .
$$

Since

$$
g_{m}^{\prime}(r)=(2 m+1) r^{m-1}\left(r^{m+1}-(m+1) r+m\right)>0,
$$

we have $g_{m}(r)>g_{m}(1)=0$, which implies

$$
m\left(r^{m}+1\right)\left(r^{m+1}-1\right)<(m+1)\left(r^{m+1}+1\right)\left(r^{m}-1\right) .
$$

We can prove similarly

$$
m\left(r^{m}+1\right)\left(r^{m+1}-1\right)>(m+1)\left(r^{m+1}+1\right)\left(r^{m}-1\right)
$$

for the case $0<r<1$.

(iii) Since we have

$$
\lim _{m \rightarrow \infty} m\left(t^{1 / m}-1\right)=\lim _{p \rightarrow 0} \frac{t^{p}-1}{p}=\log t
$$

for $t>0$ and $t \neq 1$, we have

$$
\lim _{m \rightarrow \infty} \alpha_{m}(t)=L(t, 1), \quad \lim _{m \rightarrow \infty} \beta_{m}(t)=L(t, 1) .
$$

The arithmetic-geometric mean inequality proves $\alpha_{m}(t)<\beta_{m}(t)$ for $t>0$ and $t \neq 1$. 
As standard bounds of the Riemann sum for the integral $\int_{0}^{1} t^{v} d v$, we have

$$
\gamma_{m}(t)<\int_{0}^{1} t^{\nu} d \nu<\delta_{m}(t) \quad(0<t<1)
$$

and

$$
\delta_{m}(t)<\int_{0}^{1} t^{v} d v<\gamma_{m}(t) \quad(t>1)
$$

where

$$
\gamma_{m}(t) \equiv \frac{1}{m} \sum_{k=1}^{m} t^{k / m}, \quad \delta_{m}(t) \equiv \frac{1}{m} \sum_{k=0}^{m-1} t^{k / m}
$$

Then we have the following relations.

\section{Proposition 6.2}

(i) For $0<t<1$, we have $\alpha_{m}(t)>\gamma_{m}(t)$ and $\beta_{m}(t)>\delta_{m}(t)$.

(ii) For $t>1$, we have $\alpha_{m}(t)>\delta_{m}(t)$ and $\beta_{m}(t)<\gamma_{m}(t)$.

\section{Proof}

(i) For the case $0<t<1$, the following calculations show assertion:

$$
\alpha_{m}(t)-\gamma_{m}(t)=\frac{(t-1)\left(t^{1 /(2 m)}-t^{1 / m}\right)}{m\left(t^{1 / m}-1\right)}>0, \quad \beta_{m}(t)-\delta_{m}(t)=\frac{t+1}{2 m}>0 .
$$

(ii) For the case $t>1$, the following calculations show assertion:

$$
\alpha_{m}(t)-\delta_{m}(t)=\frac{t+t^{1 /(2 m)}}{m\left(t^{1 /(2 m)}+1\right)}>0, \quad \beta_{m}(t)-\gamma_{m}(t)=\frac{1-t}{2 m}<0
$$

\section{Competing interests}

The authors declare that they have no competing interests.

\section{Authors' contributions}

The work presented here was carried out in collaboration between all authors. The study was initiated by SF and the manuscript was written by SF. SF also played the role of the corresponding author. The proof of Lemma 1.1 and the first equality of Eq. (16) in the proof of Lemma 3.3 were given by KY. With the exception of them, the proofs of all results were given by SF. All authors have contributed to, checked and approved the manuscript.

\section{Author details}

'Department of Information Science, College of Humanities and Sciences, Nihon University, 3-25-40, Sakurajyousui, Setagaya-ku, Tokyo, 156-8550, Japan. ${ }^{2}$ Division of Applied Mathematical Science, Graduate School of Science and Engineering, Yamaguchi University, 2-16-1, Tokiwadai, Ube City, 755-0811, Japan.

\section{Acknowledgements}

The author (SF) was partially supported by JSPS KAKENHI Grant No. 24540146. The author (KY) was also partially supported by JSPS KAKENHI Grant No. 23540208. 


\section{References}

1. Lin, T-P: The power mean and the logarithmic mean. Am. Math. Mon. 81, 879-883 (1974)

2. Zou, L: Matrix versions of the classical Pólya inequality. ScienceAsia 39, 204-207 (2013)

3. Niculescu, CP, Person, LE: Convex Functions and Their Applications: A Contemporary Approach. CMS Books in Mathematics, vol. 23. Springer, New York (2006)

4. Lin, M: Power-logarithmic mean inequality for Frobenius norm. Personal communication

5. Hiai, F, Kosaki, H: Comparison of various means for operators. J. Funct. Anal. 163, 300-323 (1999)

6. Hiai, F, Kosaki, H: Means of Hilbert Space Operators. Lecture Notes in Mathematics, vol. 1820. Springer, Berlin (2003)

7. Hiai, F: Matrix analysis: matrix monotone functions, matrix means, and majorization. Interdiscip. Inf. Sci. 16, 139-248 (2010)

8. Kubo, F, Ando, T: Means of positive operators. Math. Ann. 264, 205-224 (1980)

10.1186/1029-242X-2013-535

Cite this article as: Furuichi and Yanagi: Bounds of the logarithmic mean. Journal of Inequalities and Applications 2013, 2013:535

\section{Submit your manuscript to a SpringerOpen ${ }^{\circ}$ journal and benefit from:}

- Convenient online submission

- Rigorous peer review

- Immediate publication on acceptance

- Open access: articles freely available online

- High visibility within the field

- Retaining the copyright to your article 